\title{
Horizontal Lloyd mirror patterns from straight and curved nonlinear internal waves
}

\author{
K. G. McMahon, ${ }^{\text {a) }}$ L. K. Reilly-Raska, ${ }^{\text {b) }}$ and W. L. Siegmann \\ Mathematical Sciences Department, Rensselaer Polytechnic Institute, Troy, New York 12180 \\ James F. Lynch and T. F. Duda
Applied Ocean Physics \& Engineering Department, Woods Hole Oceanographic Institution, Woods Hole,
Massachusetts 02543
}

(Received 9 December 2010; revised 14 July 2011; accepted 19 July 2011)

\begin{abstract}
Experimental observations and theoretical studies show that nonlinear internal waves occur widely in shallow water and cause acoustic propagation effects including ducting and mode coupling. Horizontal ducting results when acoustic modes travel between internal wave fronts that form waveguide boundaries. For small grazing angles between a mode trajectory and a front, an interference pattern may arise that is a horizontal Lloyd mirror pattern. An analytic description for this feature is provided along with comparisons between results from the formulated model predicting a horizontal Lloyd mirror pattern and an adiabatic mode parabolic equation. Different waveguide models are considered, including boxcar and jump sound speed profiles where change in sound speed is assumed $12 \mathrm{~m} / \mathrm{s}$. Modifications to the model are made to include multiple and moving fronts. The focus of this analysis is on different front locations relative to the source as well as on the number of fronts and their curvatures and speeds. Curvature influences mode incidence angles and thereby changes the interference patterns. For sources oriented so that the front appears concave, the areas with interference patterns shrink as curvature increases, while convexly oriented fronts cause patterns to expand. (C) 2012 Acoustical Society of America. [DOI: 10.1121/1.3666004]
\end{abstract}

PACS number(s): 43.30.Bp, 43.30.Zk [JAC]

Pages: $1689-1700$

\section{INTRODUCTION}

Nonlinear internal gravity waves (NIWs) in coastal waters can strongly influence acoustic propagation. ${ }^{1}$ Although NIWs occur worldwide, ${ }^{2}$ they are ubiquitous in shallow water and vary considerably in their characteristics, ${ }^{3}$ and their acoustic effects are of particular experimental interest. The waves are often observed from late spring through early fall in periods of sharp stratification. ${ }^{2}$ In a well know process, nonlinear internal waves cause sound speed variability by vertically displacing water parcels, and the vertical structure of these internal waves is well described using a few low order baroclinic modes. The NIWs produce relatively large isopycnal displacements ${ }^{4}$ (10-30 m or more), and corresponding large changes in the water sound speed profile. The consequences of acoustic scattering by NIWs can be significant changes in the vertical acoustic energy distribution, particularly for low frequency $(<1 \mathrm{kHz}$,$) signals. { }^{5}$

A review of some results from the Shallow Water ' 06 experiment describes features of propagation through NIWs. ${ }^{6}$ These include two-dimensional (2-D) propagation across NIW fronts that cause fluctuations of sound intensity arising from mode coupling. ${ }^{7}$ Such intensity changes were confirmed in 3-D NIW environments in which acoustic calculations were performed without azimuthal coupling

\footnotetext{
a) Author to whom correspondence should be addressed. Electronic mail: mcmahk3@rpi.edu

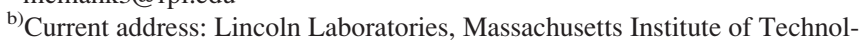
ogy, 244 Wood Street, Lexington, MA 02420.
}

$(\mathrm{N} \times 2-\mathrm{D}) .{ }^{8}$ The NIW packets produced strong intensity fluctuations when the angle between acoustic propagation direction and NIW fronts is larger than about $20 \mathrm{deg}$. For smaller angles, and especially when the propagation is near parallel to the fronts, calculations show intensity focusing and defocusing. ${ }^{9}$ This strong effect was observed by analysis of data collected during the 1995 SWARM experiment. ${ }^{10,11}$ Fully 3 -D propagation calculations ${ }^{12}$ also show this behavior and suggest where azimuthal coupling may be important. A summary of propagation mechanisms as a function of angle between acoustic direction and NIW front is provided in Ref. 13.

At small angles between propagation directions and NIW fronts, horizontal refraction effects may lead to distinctive interference patterns or striations in horizontal transmission loss (TL), especially at lower frequencies. These striations were hypothesized to arise from interference between a direct propagation path and a path refracted by the NIW front. The pattern was called a Horizontal Lloyd mirror (HLM) in Ref. 1 because of the correspondence to the classical Lloyd mirror in a vertical plane with a surfacereflected path. The classical Lloyd mirror has a long history and is relevant to many shallow water applications. ${ }^{14}$ The HLM patterns are of considerable interest because they have been observed, for the first time, in experimental data taken during Shallow Water ' $06^{15}$ and discussed in experimental observations in Ref. 16. Moreover, their importance has been conjectured for containing information that potentially may be extracted for characterizing NIW features. ${ }^{1}$ The objective of this study is to explore properties of HLM 
interference patterns and their dependence on environmental and internal wave parameters. Different representations of NIWs are considered and compared to find one that is both convenient and generally faithful to the widely used Korteweg-de Vries (KdV) solitons. The selected sound speed models are benchmarked with the Adiabatic Mode Parabolic Equation (AMPE). ${ }^{17}$ With this model established, extensions are made to incorporate multiple waves because solitons are known to travel in trains. Fronts exhibiting different degrees of curvature are observed experimentally in synthetic aperture radar (SAR) images from many experiments, ${ }^{2}$ so this property may be expected to be particularly important. As a result, both straight and curved internal wave fronts are considered.

The paper is structured as follows. The next section describes three NIW approximations, a $\operatorname{sech}^{2}$, a boxcar shape, and single sound speed jump. A model for NIW interactions is formulated and describes full-field acoustic calculations used for AMPE. Section III formulates the horizontal Lloyd mirror model using Huygens' Principle and provides convenient expressions for later use. Extensions are made for multiple and moving NIWs. A revised formulation for the horizontal Lloyd mirror for curved NIW fronts, again using Huygens' Principle, appears in Sec. IV. Dependence of the interference pattern on parameters such as wave spacing and speed, as well as frontal curvature is discussed in Sec. V. The final section discusses results and conclusions as well as future work.

\section{ENVIRONMENTAL MODEL AND PROPAGATION CALCULATIONS}

\section{A. Environmental formulation}

The ocean regional environment used in this study has properties based on the New Jersey shelf, including trains of NIWs observed and recorded in the 1995 SWARM $^{18}$ and Shallow Water 06 experiments. ${ }^{6}$ A range independent background water sound speed profile $c_{0}(z)$ from the SWARM experiment appears as the solid curve in Fig. 1(a). It suggests that $c_{0}(z)$ has two layers, so a simplified profile ${ }^{10}$ shown with dashed lines in Fig. 1(a) is used. The upper layer sound speed decreases with constant gradient to depth $45 \mathrm{~m}$, from surface value of 1534 to $1484 \mathrm{~m} / \mathrm{s}$, and the lower layer is isospeed. The bottom bathymetry is approximated as flat with depth $H=71 \mathrm{~m}$. The sediment sound speed has a $20 \mathrm{~m}$ transition layer from 1600 to $1650 \mathrm{~m} / \mathrm{s}$, with an isospeed basement of $1650 \mathrm{~m} / \mathrm{s}$ continuing to $1000 \mathrm{~m}$. The bottom is simplified to neglect attenuation, so that the focus of the study can be on water column effects. The canonical sound speed environment used in this study is intended to exemplify important ocean acoustic propagation physics. Clearly the detailed acoustic response relevant to any particular experiment will depend on the specific environment for that experiment. Other water sound speed profiles lead to acoustic responses that could be concentrated in different mode numbers. ${ }^{19}$

NIWs give rise to vertical isopycnal displacements, $\eta$, which can be written using Eq. (1). ${ }^{3}$ A cylindrical coordinate system $(r, \theta, z)$ is used, where $z=0$ and $H$ are the ocean surfaces, $r$ is range, and $\theta$ is azimuth. Separating variables with $F(r, \theta)$ as the horizontal displacement,

$$
\eta(r, \theta, z)=F(r, \theta) \hat{\eta}(z)
$$

leads to a boundary value problem for the internal wave modes $\hat{\eta}_{j}$ :

$$
\begin{aligned}
& \frac{\mathrm{d}^{2} \hat{\eta}_{j}}{\mathrm{~d} z^{2}}+\kappa_{j}^{2}\left(\frac{\bar{N}^{2}(z)-\omega_{j}^{2}}{\omega_{j}^{2}-f_{i n}^{2}}\right) \hat{\eta}_{j}=0, \\
& \hat{\eta}_{j}(0)=\hat{\eta}_{j}(H)=0,
\end{aligned}
$$

where the linear internal wave mode, $\hat{\eta}$, can be calculated assuming a flat ocean bottom with no background currents. In Eq. (2) $\bar{N}(z)$ is buoyancy frequency, $f_{i n}$ is local inertial frequency, $\omega_{j}$ are eigenvalues, and $\kappa_{j}$ are the horizontal internal wave wavenumbers. To incorporate internal wave effects, only the first internal wave mode $\eta_{1}(z)$ (Ref. 20) is considered. Higher modes may be considered once the model is established because they are relatively easy to treat. The first modal shape is simplified as having two layers, each approximated linearly. ${ }^{5}$ In deep water, the ratio of internal wave amplitudes to water depth is small with the horizontal motion expressed as a plane wave. However, in shallow water this ratio can be much larger, and soliton solutions of the nonlinear $\mathrm{KdV}$ equation are widely used as models. ${ }^{2,21}$ Following Refs. 5 and 21, the horizontal isopycnal depressions from a series of $N$ NIWs are

$$
F(r, \theta)=\sum_{i=1}^{N} A_{i} \operatorname{sech}^{2}\left(\frac{y(r, \theta)-y_{i}-V_{i} t}{\Lambda_{i}}\right)
$$

where $A_{i}, \Lambda_{i}, y_{i}$, and $V_{i}$ are the amplitude, width, relative position, and speed of a planar wavefront which travels in the direction of the $y$ axis.

The most significant acoustic activity usually occurs near the lead internal wave in an internal wave train (typically the lead wave is the largest). ${ }^{5,22}$ Refraction and reflection effects determine variations of the acoustic field. These mechanisms are controlled by the angle between the directions of acoustic propagation and internal wavefronts. Significant refraction near the NIW occurs when this angle is small; that is to say, the acoustic propagation direction is along the NIW front. The spacing between waves is assumed wide enough so that propagation contributions arise from the lead internal wave within a circular region of interest [see Fig. 1(b)]. The lead wave is spatially coherent and selfhealing to perturbations, so it does not change significantly in height or width. ${ }^{22}$ Consequently, the wave preserves its shape as it moves relative to the acoustic source, at the center of the region, and calculations are illustrated for various NIW locations [choices of $t$ in Eq. (4)]. Thermocline displacements based on this shape are shown in Fig. 1(c), where the maximum range is $15 \mathrm{~km}$ and cross range distance is $800 \mathrm{~m}$. Amplitude $A_{1}=10 \mathrm{~m}$ and wavelength $\Lambda_{1}=270 \mathrm{~m}$ lead to an average sound speed increase of about $12 \mathrm{~m} / \mathrm{s}$, using Eq. (4) and following Ref. 21. For comparison, an 


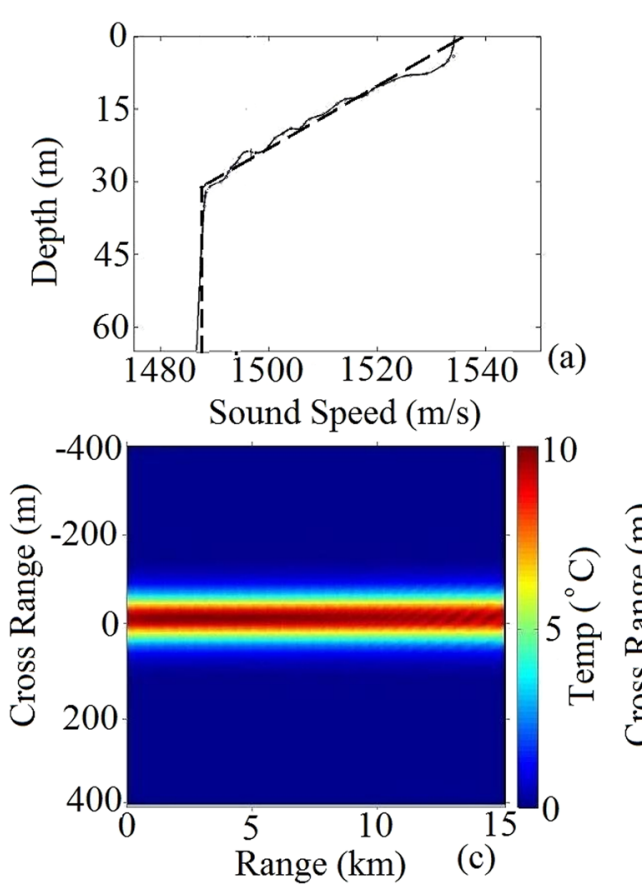

(a)

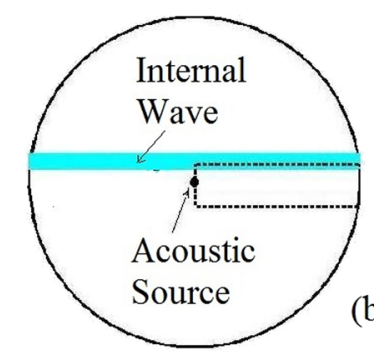

(b)

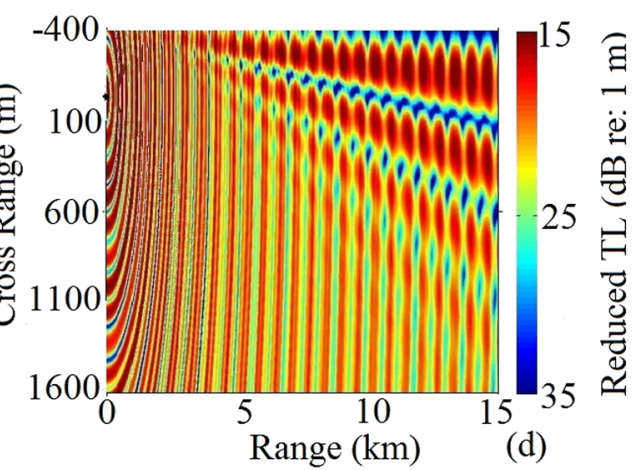

FIG. 1. (Color online) Nonlinear internal wave environment. (a) Background water sound speed profile based on the SWARM region is shown in the solid line. Dashed lines represent a two-part linear approximation to the SWARM profile. (b) Horizontal schematic with $\mathrm{CW}$ source located at the center of a circular ocean region (radius, $15 \mathrm{~km}$ ). Environmental features and calculations are displayed for the $15 \mathrm{~km} \times 2 \mathrm{~km}$ box enclosed by the black dashed line. (c) Thermocline for NIW example from $0^{\circ} \mathrm{C}$ to $10^{\circ} \mathrm{C}$. (d) Reduced transmission loss ( $\mathrm{dB}$ re: $1 \mathrm{~m})$ contours in a horizontal plane for the boxed region shown in (b). Four-mode calculation at $55 \mathrm{~Hz}$ from AMPE. The internal wave in (c) lies along upper image edge, $400 \mathrm{~m}$ from the source. The interference pattern of energy refracted by the internal wave is multi-modal. example with $A_{1}=20$ and twice the sound speed increase is shown.

\section{B. Acoustic propagation computations}

Relatively slow horizontal changes justify the assumption of adiabatic propagation where conditions are not close to mode cutoffs. Also, forward scattering is an excellent approximation for these environments. As a result TL calculations are preformed using AMPE, ${ }^{17}$ which assumes no coupling between acoustic normal modes. The pressure field is represented as a finite sum of $M$ propagating modes,

$$
P(r, z, \theta)=\sum_{m=1}^{M}\left[k_{m}(r, \theta)\right]^{1 / 2} u_{m}(r, \theta) \psi_{m}(z ; r, \theta) .
$$

The horizontal wavenumber $k_{m}$ varies slowly in $(r, \theta)$, which permits energy conservation to be satisfied conveniently. The local acoustic modes $\psi_{m}$ incorporate slow horizontal variation from NIWs, and the amplitude $u_{m}$ is found from a $\mathrm{PE}$ in range $\theta$. Sound speed changes from NIWs are discretized on a horizontal grid, and $k_{m}$ and $\psi_{m}$ are calculated at each grid point. The normal mode code COUPLE (Ref. 23) is used to calculate mode shapes and wavenumbers with the propagation range divided into range-independent subregions and a strong absorption layer imposed in the bottom $100 \mathrm{~m}$.

Because a Lloyd mirror pattern should arise where propagation is nearly parallel to waveguide boundaries, calculations are shown in the region of Fig. 1(a) enclosed by the black dashed line. Different spacings $s$ between the NIW and the acoustic source measure the progression of the internal wave as it passes over the source. A frequency of $f=55 \mathrm{~Hz}$ is used because it is within the range where strong NIW activity was observed in the SWARM experiment. ${ }^{10}$ For this frequency, the waveguide supports four propagating modes. Figure 1(d) shows TL reduced by cylindrical spreading $(r)$,

$$
T L=-10 \log \left(\frac{I}{I_{0}}\right)+10 \log (r),
$$

with a reference intensity $I_{0}$, for the NIW model in Eq. (4), where $s=400 \mathrm{~m}, N=1$, and $A_{1}=10 \mathrm{~m}$. Although the modal interference from the four modes is clearly visible, the pattern created by horizontal refraction from the NIW is somewhat obscured. To focus on structure of the patterns, subsequent calculations use only the first propagating acoustic mode. The TL contours from AMPE for this case are shown in Figs. 2(a) and 2(b), where the distances $s$ are 400 and $800 \mathrm{~m}$ and the cross range of $1.8 \mathrm{~km}$ corresponds to part of the boxed region in Fig. 1(b). Contours in all plots are shown over a $10 \mathrm{~dB}$ dynamic range. Figures $1(\mathrm{~d}), 2(\mathrm{a})$, and 2(b) show that a single mode permits focus on the basic interference patterns. However, a frequency that supports multiple modes is used because often one mode dominates. This allows better extraction of interference pattern properties and also the possibility to extend calculations to multiple modes.

Two modifications of the $\operatorname{sech}^{2}$ NIW environment are considered. The first approximates the $\operatorname{sech}^{2}$ shape by a boxcar consisting of two sound speed jumps as suggested in Fig. 2(c). This model assumes no transmission, and strong interference patterns where incident angles upon the internal wave reflector are shallow. In this case, in the direction of sound speed variation (that is, internal wave propagation), the acoustic wavelengths have longer projections. These long wavelengths move rapidly over large distances, so that small sound speed changes have little effect on them, and the boxcar approximation is appropriate. The magnitudes of the sound speed jumps do not directly correspond to the 


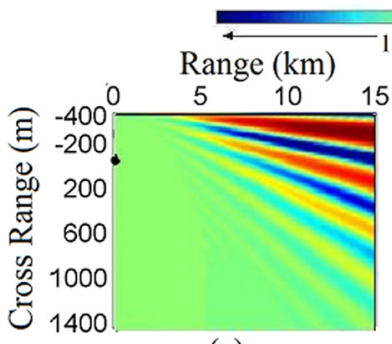

(a)

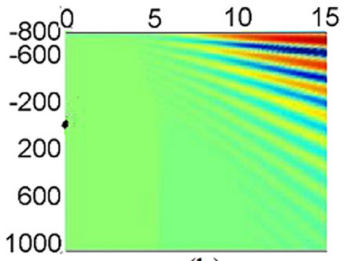

(b)

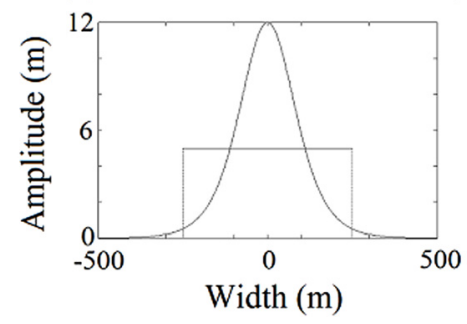

(c)

FIG. 2. (Color online) Reduced TL (dB re: $1 \mathrm{~m}$ ) contours in the horizontal from AMPE using one mode in the NIW environment. The internal wave lies along upper image edge, and source is at left boundary (black dot). Distance between internal wave acoustic source is (a) $400 \mathrm{~m}$, and (b) $800 \mathrm{~m}$. Range is $15 \mathrm{~km}$ and cross range is $1800 \mathrm{~m}$. (c) Boxcar and $\mathrm{sech}^{2}$ representations of an NIW. Boxcar width and height are chosen so that its area is about the same as the $\operatorname{sech}^{2}$.

maximum change in sound speed across the $\operatorname{sech}^{2}$. Ways to select the jump magnitude include the value at the point of maximum $\operatorname{sech}^{2}$ slope or from matching the areas under the $\mathrm{sech}^{2}$ and boxcar. The latter is used, and Fig. 2(c) sketches the two wave representations. The boxcar area depends on width and amplitude, so each must be chosen to match the $\mathrm{sech}^{2}$ area. A jump of $5 \mathrm{~m} / \mathrm{s}$ (the $1 / e$ point of the $12 \mathrm{~m} / \mathrm{s}$ sound speed variation) and a width of $500 \mathrm{~m}$ are chosen, accounting for 94 percent of the $\operatorname{sech}^{2}$ area. At the first jump interface of the boxcar, the sound speed increases, causing mainly reflection at shallow angles and relatively little transmission. At the second interface, only minor reflections occur because the sound speed decreases. Therefore, the second modification is to approximate the thermocline displacement as a single jump in sound speed between the two isospeed regions. Calculations to follow use these simplifications and focus on the strength of the associated NIW sound speed jump, the number of wavefronts and their motion as well as the source to front separation and frontal curvature.

\section{HORIZONTAL LLOYD MIRROR}

In this section, the HLM is formulated using Huygens' Principle. The classical Lloyd mirror for propagation in a vertical plane takes pressure as the sum of a direct and ocean surface-reflected wave. ${ }^{14}$ In the HLM, an adiabatic mode propagates along horizontal rays, in a horizontal plane. ${ }^{24}$ It is appropriate to assume that any adiabatic vertical mode retains its energy during propagation for sufficiently slow azimuthal changes.

To develop the HLM model, this vertical geometry is rotated to the horizontal plane, shown in Fig. 3(a). The parameter $\theta_{1}$, not associated with the angle $\theta$ in Eq. (5), is the angle between the acoustic propagation direction and NIW crests. The straight NIW reflector is taken along $y=y_{F}$. The source $\mathrm{S}$ is positioned at $(0,0)$ and the receiver at $(L, d)$. The direct and reflected paths to the receiver are $r_{0}$ and $r_{1}+r_{2}$, where

$$
r_{1}\left(\theta_{1}\right)=\sqrt{x^{2}+y_{F}^{2}}=\frac{x}{\cos \theta_{1}}
$$

and,

$$
r_{2}\left(\theta_{1}\right)=\sqrt{d^{2}+(L-x)^{2}}=\frac{L-x}{\cos \theta_{2}\left(\theta_{1}\right)} .
$$

The pressure $P(x, y, z)$ satisfies the 3-D Helmholtz equation for a source located at $\left(x_{s}, y_{s}, z_{s}\right)$,

$$
\begin{aligned}
\nabla^{2} P & +\frac{1}{\rho} \frac{\partial}{\partial z}\left(\rho \frac{\partial P}{\partial z}\right)+k^{2}(z ; x, y) P \\
= & -4 \pi \delta\left(x-x_{s}\right) \delta\left(y-y_{s}\right) \delta\left(z-z_{s}\right)
\end{aligned}
$$

A separable solution of Eq. (9) is

$$
P(x, y, z)=\Gamma_{m}(x, y) \psi_{m}(z ; x, y)
$$

where $\Gamma_{m}(x, y)$ is the modal amplitude and $\psi_{m}(z ; x, y)$ is the $m$ th modal depth function. Because from Sec. II B a single mode is considered, the mode number subscript $m$ is dropped so that the modal amplitude is governed by

$$
\nabla^{2} \Gamma+k_{H}^{2} \Gamma=-4 \pi \frac{\psi\left(z_{s} ; x_{s}, y_{s}\right)}{\rho\left(z_{s}\right)} \delta\left(x-x_{s}\right) \delta\left(y-y_{s}\right),
$$

where $k_{H}=\sqrt{k^{2}-k_{z}^{2}(z ; x, y)}$ is the horizontal wavenumber. The quantity $k_{H}$ has a slow variation in $x$ and $y$, but in the present model, it is constant inside and outside of the duct. In cylindrical coordinates, the axisymmteric solution to Eq. (11) is

$$
\Gamma(r)=i \pi \frac{\psi\left(z_{s} ; r_{s}\right)}{\rho\left(z_{s}\right)} H_{0}^{(1)}\left(k_{H}\left|r-r_{s}\right|\right),
$$

which yields solutions of the form

$$
\Gamma(r) \sim q \frac{e^{i k_{H}\left|r-r_{s}\right|}}{\sqrt{k_{H}\left|r-r_{s}\right|}},
$$

in the far-field approximation. The variable $r$ is the radial coordinate, $r_{s}$ the source position, and the constant $q=i \sqrt{2 \pi} e^{-i \pi / 4} \psi\left(z_{s} ; r_{s}\right) / \rho\left(z_{s}\right)$.

\section{A. Huygens' principle formulation}

Huygens' method is used to formulate an expression for a reflected wave at low grazing angles. Each wave is treated as a different source with diminished strength due to the amplitude decrease $1 / \sqrt{k_{H} r_{1}}$ along the propagation path, and the obliquity factor and the Kirchoff Integral Theorem are incorporated. $^{25}$ There are at least two other approaches to find this amplitude, including the image method and plane wave decomposition. ${ }^{26}$ 


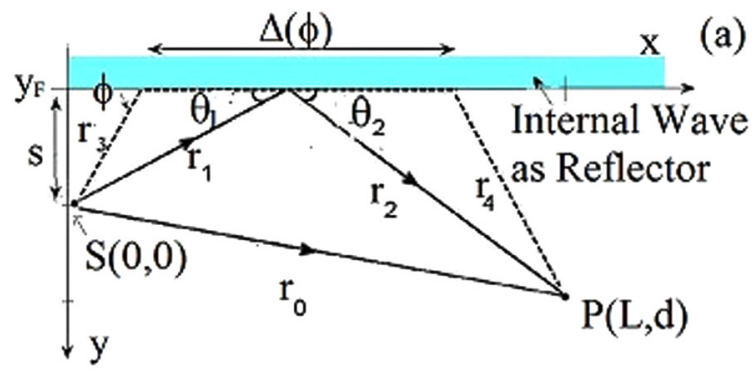

(a)

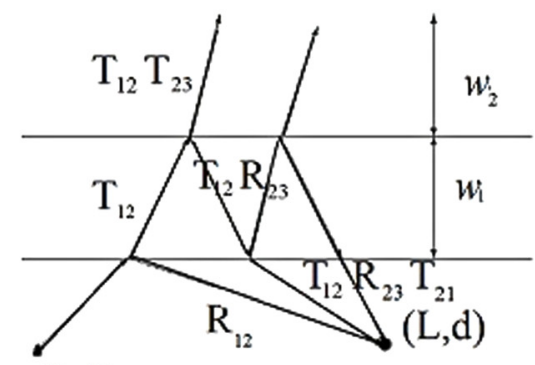

$(0,0)$

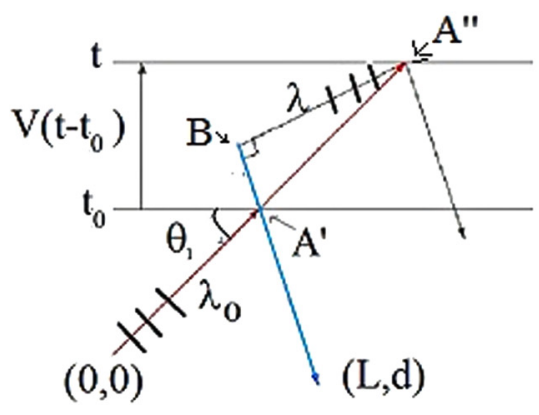

(c)

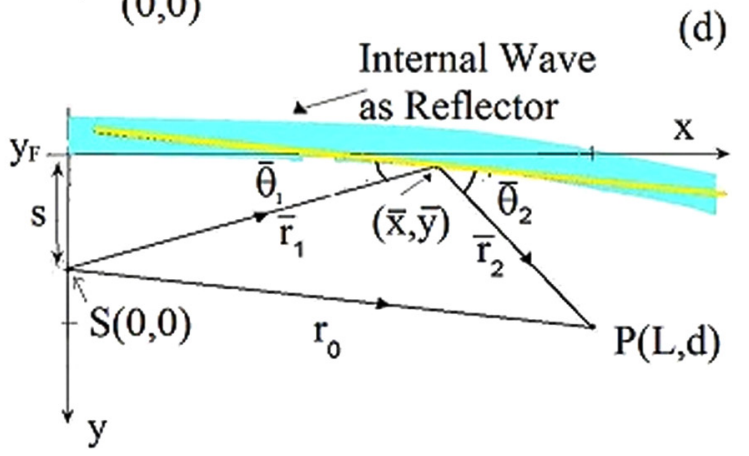

FIG. 3. (Color online) (a) Horizontal Lloyd mirror geometry with NIW modeled as a sound speed jump. Acoustic source $S$ is located at the origin and pressure $P$ is received at $(L, d)$. Propagation paths shown for a direct wave $r_{0}$ and a reflected wave $r_{1}$ and $r_{2}$. For incident angles $\phi$ near critical, beam displacement $\Delta(\phi)$ occurs. (b) Schematic for multiple wavefronts incorporating dominant secondary reflections and transmissions from additional fronts. The value $w_{j}$ represents the width of layer $j$. Transmission $T_{i j}$ and reflection $R_{i j}$ coefficients are from region $i$ to region $j$. (c) Schematic of NIW front at initial time $t_{0}$ and a later time $t$ after the wave moves with constant speed $V$. The incident sound path from $(0,0)$ to $A^{\prime}$ has wavelength $\lambda_{0}=c / f_{0}$. The sound reflects off the front at $t_{0}$ and at $t$. The projected wavelength of reflected sound $\lambda$ is the shortest distance between the reflected paths. (d) Horizontal Lloyd mirror geometry where a curved nonlinear internal wave is modeled as a sound speed jump. The front shown corresponds to the plus root of Eq. (44). Propagation paths are shown for a direct wave $\left(r_{0}\right)$ and a reflected wave $\left(\bar{r}_{1}, \bar{r}_{2}\right)$. The line tangent to the front at the stationary point $\infty$ and $\bar{\theta}_{1}$ and $\bar{\theta}_{2}$ are the angles between reflected rays and this tangent.

Huygens' formulation requires finding amplitudes and phases of the sources on the NIW front, which when integrated comprise the reflected wave at the receiver. Reciprocity of the Green's functions is used with a source at $(L, d)$ for calculations. The free space Green's function $G(x, y)$ that satisfies Eq. (11) with $\left(x_{s}, y_{s}\right)=(L, d)$ is used with the modal amplitude $\Gamma(x, y)$ where $\left(x_{s}, y_{s}\right)=(0,0)$. For a volume $\Omega$ and surface $\hat{S}$, Green's Identity is

$$
\int_{\Omega} \Gamma \nabla^{2} G-G \nabla^{2} \Gamma \mathrm{d} \Omega=\int_{\hat{S}} \Gamma \frac{\partial G}{\partial \hat{n}}-G \frac{\partial G}{\partial \hat{n}} d \hat{S}
$$

where $\hat{n}$ is the outward pointing unit normal to the wavefront. Substituting $G(x, y ; L, d)$ and $\Gamma(x, y ; 0,0)$, which have the form of Eq. (13), into Eq. (14) and solving for $\Gamma(L, d)$ gives

$$
\begin{aligned}
\Gamma(L, d)= & \frac{\psi\left(z_{s} ; 0,0\right)}{\psi\left(z_{s} ; L, d\right)} G(0,0 ; L, d) \\
& -\frac{\rho\left(z_{s}\right)}{4 \pi \psi\left(z_{s} ; L, d\right)} \int_{s} \Gamma \frac{\partial G}{\partial \hat{n}}-G \frac{\partial \Gamma}{\partial \hat{n}} d \hat{S} .
\end{aligned}
$$

The definition of $q$ implies that

$$
\frac{\psi\left(z_{s} ; 0,0\right)}{\psi\left(z_{s} ; L, d\right)} G(0,0) \sim q \frac{e^{i k_{H} r_{0}}}{\sqrt{k_{H} r_{0}}} .
$$

The integral portion of Eq. (15), denoted as $\Gamma_{R}(x, y)$, is the Kirchoff Integral, and the Kirchoff boundary conditions imply that the only portion of $\hat{S}$ that contributes is along the wavefront $y=y_{F}$. Both amplitude and Green's functions are evaluated at $y=y_{F}$ and have solutions like Eq. (13) with $\left|r-r_{s}\right|$ replaced by $r_{1}$ and $r_{2}$. The obliquity factor $C\left(\theta_{1}, \theta_{2}\right)$ converts the normal derivative terms in Eq. (15) to derivatives with respect to $r_{1}$ or $r_{2}$. This factor is an average of cosines of the normals with respect to the horizontal for the incident and reflected angles,

$$
C\left(\theta_{1}, \theta_{2}\right)=\frac{\cos \left(\frac{\pi}{2}-\theta_{1}\right)+\cos \left(\frac{\pi}{2}-\theta_{2}\right)}{2},
$$

and accounts for the angular tilt of the source. Finally, the boundary condition for $\Gamma(x, y ; 0,0)$ includes the coefficient $R\left(\theta_{1}\right)$ to account for reflection off the wavefront surface and is discussed later.

From Eqs. (15) and (17), the amplitude of the reflected wave is given by

$$
\Gamma_{R}(L, d) \sim i k_{H} \frac{\psi\left(z_{s} ; 0,0\right)}{\rho\left(z_{s}\right)} \int_{0}^{\infty} R\left(\theta_{1}\right) C\left(\theta_{1} \theta_{2}\right) \frac{e^{i k_{H}\left(r_{1}+r_{2}\right)}}{\sqrt{k_{H}^{2} r_{1} r_{2}}} d x,
$$

where integration in $x$ implies summation of all possible sources along the $x$ axis. Stationary phase is employed to evaluate the integral expression in Eq. (18) for large $k_{H}$. Using Eqs. (7) and (8), the phase is

$$
\phi(x, y)=k_{H}\left(\frac{x}{\cos \theta_{1}(x)}+\frac{L-x}{\cos \theta_{2}(x, y)}\right) .
$$


The major contribution from stationary phase occurs when $\cos \theta_{1}=\cos \theta_{2}$, or $\theta_{1}=\theta_{2}=\theta_{s}$, giving

$$
\Gamma_{R}(L, d) \sim R\left(\theta_{s}\right) \frac{i \sqrt{2 \pi} \psi\left(z_{s} ; 0,0\right)}{\rho\left(z_{s}\right)} \frac{e^{i k_{H}\left(r_{1}+r_{2}\right)-i \pi / 4}}{\sqrt{k_{H}\left(r_{1}+r_{2}\right)}} .
$$

Using the definition of $q$ and Eqs. (16) and (20) gives the amplitude as

$$
\Gamma(L, d)=q\left(\frac{e^{i k_{H} r_{0}}}{\sqrt{k_{H} r_{0}}}+R\left(\theta_{1}\right) \frac{e^{i k_{H}\left(r_{1}+r_{2}\right)}}{\sqrt{k_{H}\left(r_{1}+r_{2}\right)}}\right) .
$$

Internal wave effects alter the reflection coefficient from the traditional Rayleigh formula, which involves the index of refraction $n=\hat{k} / k$. A variation of this reflection coefficient appears in Ref. 1, which is called the ESME reflection coefficient. It is expressed as

$$
R\left(\theta_{s}\right)=\frac{\sin \theta_{s}-i \sqrt{\cos ^{2} \theta_{s}-v^{2}}}{\sin \theta_{s}+i \sqrt{\cos ^{2} \theta_{s}-v^{2}}},
$$

where $v_{1}=2 \pi f / k_{H}$ and $v_{2}=2 \pi f / \hat{k}_{H}$ are the horizontal phase velocities for frequency $f$ and the horizontal wavenumber in the upper medium $\hat{k}_{H}$. The ratio of horizontal phase velocities $v=v_{1} / v_{2}$ may be different from $n$ for larger angles. ${ }^{1}$ In this case, propagation at small incidence angles and low acoustic modes, wavenumbers are close to that of the ocean region and to each other, so a ratio of phase velocities well approximates the index of refraction.

The reflection coefficient permits only wave reflection for incident angles smaller than the critical angle. Thus the wavefront is not a perfect reflector, and $R \neq 1$ for all propagation angles. The critical angle is defined as

$$
\varphi=\cos ^{-1} \nu .
$$

Using Eqs. (7) and (8) implies

$$
v \geq \frac{y_{F}}{\sqrt{x^{2}+y_{F}^{2}}},
$$

and

$$
v \geq \frac{d}{\sqrt{(L-x)^{2}+d^{2}}},
$$

for the $\left|R\left(\theta_{s}\right)\right| \leq 1$. Combining the conditions yields an equation which predicts locations $d$ in terms of $y_{F}$ and $L$ for which to expect an HLM interference pattern:

$$
d\left(L, y_{F}\right) \leq L \sqrt{\frac{v^{2}}{1-v^{2}}}-y_{F}
$$

The constraint Eq. (26) assumes that the magnitude of the reflection coefficient decreases from one sharply and no reflection occurs outside the constrained region. Although the transition is rapid it is not immediate, so Eq. (26) gives the region in which a strong HLM pattern occurs.

For the selected sound speed values of $c=1500$ and $\hat{c}=1512 \mathrm{~m} / \mathrm{s}$, the critical angle is about $7 \mathrm{deg}$. This particular sound speed jump restricts the $d, y_{F}$, and $L$ values giving a HLM pattern according to Eq. (26). For example, when $s=400 \mathrm{~m}$ and $L=15 \mathrm{~km}$, the maximum value of $d$ is about $1428 \mathrm{~m}$. Choosing $c$ as above and $\hat{c}=1524 \mathrm{~m} / \mathrm{s}$, however, gives a critical angle of about $10 \mathrm{deg}$, allowing a maximum of about $d=2250 \mathrm{~m}$ for the same $y_{F}$ (or $s$ ) and $L$.

In addition to horizontal sound speed changes, the vertical sound speed profile also influences the HLM pattern. Several approximations may be used to describe the sound speed profiles found during the SW06 experiment. ${ }^{19}$ The established vertical profile gives a separate dependence of the interference pattern on mode number. The mode number changes both $k_{H}$ and the reflection coefficient, and the dominant effect on the pattern results from changes in the former. For the region of interest, the sound paths $r_{0}$ and $\left(r_{1}+r_{2}\right)$ only differ by a few percent. The major contribution in TL will therefore arise from the phase terms in Eq. (21) and from the mode number. This is seen by following the formulation in Refs. 14 and 27 for the classical Lloyd mirror model. Using $L \gg s$, the direct and reflected paths in Eq. (21) are approximated to show the magnitude of the modal amplitude. Adjacent nulls in the amplitude are spaced in cross range according to

$$
\left|d_{i}-d_{i+1}\right|=L k_{H} s\left|\sqrt{1-\left(\frac{i \pi}{k_{H} s}\right)^{2}}-\sqrt{1-\left(\frac{(i+1) \pi}{k_{H} s}\right)^{2}}\right|,
$$

for $i=0,1, \ldots$, where $i$ indexes the zeros in the magnitude of Eq. (21). Because $k_{H} s i \pi$, and an HLM pattern is only supported for ranges much greater than cross range, a binomial expansion of Eq. (27) for each internal wave mode $m$ yields

$$
\begin{aligned}
\left|d_{i}-d_{i+1}\right| & \sim L \pi^{2} \frac{2 i+1}{k_{H} s} \\
& =L \pi^{2} \frac{2 i+1}{s \sqrt{\left(\frac{2 \pi f}{c}\right)^{2}-\left(\frac{(2 m-1) \pi}{2 H}\right)^{2}}},
\end{aligned}
$$

for $m=0,1, \ldots$ As $i$ increases, so does null spacing. As mode number increases, the spacing increases and the HLM beams become wider. Equation (28) also indicates a dependence on frequency $f$ opposite to mode number dependence as $f$ increases the null spacing causes more narrow beams.

Beam displacements are known to occur at interfaces with the ocean bottom. Because sound speed changes between the water column and sediment can be large, it may be necessary to incorporate pressure disturbances that propagate along the water-sediment interface. Figure 3(a) depicts an alternate propagation path $r_{3}+r_{4}+\Delta(\phi)$ for reflected waves near an interface, ${ }^{26}$ the lateral wave. The magnitude of the displacement $\Delta(\phi)$ is determined by the incident angle and refractive index change and is largest for incident angles near critical. In calculations, Eq. (21) is used with a lateral component $\Gamma_{L}(x, y)$ for the reflected portion: ${ }^{28}$ 


$$
\Gamma_{L}(x, y) \sim \frac{8 \pi i \hat{k}_{H} e^{-i \pi / 8}}{\Gamma\left(\frac{1}{4}\right) k_{H} \sqrt{\sin (2 \phi)}\left(k_{H}\left(r_{1}+r_{2}\right) / 2\right)^{1 / 4}} \frac{e^{i k_{H}\left(r_{1}+r_{2}\right)}}{\left(r_{1}+r_{2}\right)}
$$

which is valid for $\phi-\theta_{s}<\epsilon$. The constant $\epsilon$ is chosen such that the angles are within about $2 \mathrm{deg}$ and the quantity $\Gamma\left(\frac{1}{4}\right)$ is the Gamma function. Calculations show that beam displacements were found to have negligible effects. Another approximation of the NIW sound speed change uses two regions of constant gradient with the sound speed peak change at the center of the NIW. This simplification preserves refractive effects but will not be discussed here.

\section{B. Multiple NIWs}

Ocean NIWs travel in packets with each wave having distinct amplitudes. ${ }^{2}$ Incorporating this extension into the HLM model requires modifying the reflection coefficient. A schematic is shown in Fig. 3(b), where each $R$ and $T$ represent reflection and transmission coefficients at each interface. These reflection coefficients have the same form as Eq. (22). For $l$ isospeed layers the reflection coefficients are recursively defined as ${ }^{26,27}$

$$
R_{(l-2) l}\left(\theta_{1}\right)=\frac{R_{(l-2)(l-1)}+R_{(l-1) l}}{1+R_{(l-1) l} R_{(l-2)(l-1)} e^{2 i \Phi_{l-1}}},
$$

and

$$
R_{(l-3) l}\left(\theta_{1}\right)=\frac{R_{(l-3)(l-2)}+R_{(l-2) l}}{1+R_{(l-2) l} R_{(l-3)(l-2)} e^{2 i \Phi_{l-2}}},
$$

reaching $R_{1 l}$. Equations (30) and (31) require $l \geq 3$ and $l \geq 4$. The phase $\Phi_{j}=k_{H}^{j} w_{(j-1)} \sin \theta_{j}$, for width $w_{j}$ and horizontal wavenumber $k_{H}^{j}$ of the $j$ th layer. Snell's law is satisfied at each interface, so $k_{H}^{1} \cos \theta_{1}=k_{H}^{j} \cos \theta_{j}$ for all $j$. Layers with even values of $l$ have higher sound speed values and represent NIWs. The case $l=2$ (one front) is the jump model used so far, and $l=3$ ( 2 fronts) is the boxcar representation of the $\mathrm{sech}^{2}$. Of interest are effects of different sounds speed jumps and spacings of NIWs in the train.

\section{Moving NIWs}

The formulation above assumes a fixed NIW reflector, but NIW motion relative to a stationary source implies a Doppler shift. This appears as a frequency change and is derived using geometric arguments. ${ }^{29}$ At time $t_{0}$, the wave begins to travel at a constant wave speed $V$. At time $t$, the wave has moved to $V\left(t-t_{0}\right)$ as seen in Fig. 3(c). Incident sound begins at the source and reaches $A^{\prime}$ with wavelength $\lambda_{0}$. At time $t$, sound reflects at a different point along the front. The reflected wavelength $\lambda$ is given by the shortest distance between the reflected sound paths at times $t$ and $t_{0}$. Trigonometry gives the two relations

$$
c\left(t-t_{0}\right)=\lambda_{1}+\frac{V\left(t-t_{0}\right)}{\cos \left(\pi / 2-\theta_{1}\right)}
$$

and

$$
\lambda=V\left(t-t_{0}\right) \frac{\cos \left(\pi-\theta_{1}-\theta_{2}\right)}{\cos \left(\pi / 2-\theta_{1}\right)}+c\left(t-t_{0}\right),
$$

representing path lengths from the source to $A^{\prime \prime}$ and from point $\mathrm{B}$ to the receiver. Eliminating $\left(t-t_{0}\right)$ and $\theta_{2}$ and using the relations $f=c / \lambda$ and $f_{0}=c / \lambda_{0}$ gives the modified frequency $\tilde{f}$ :

$$
\tilde{f}=f_{0}\left(\frac{1-\frac{V}{c} \sin \left(\theta_{1}\right)-\left(\frac{V}{c}\right)^{2}}{1-\left(\frac{V}{c}\right)^{2}}\right) .
$$

This change in frequency influences the horizontal wavenumber associated with the reflected portion of the modal amplitude. Substituting Eq. (34) into $k_{H}=\sqrt{k^{2}-k_{z}^{2}(z ; x, y)}$ and expanding gives the corrected horizontal wavenumber $\tilde{k}_{H}$

$$
\tilde{k}_{H} \sim k_{H}-2 \frac{k^{2}}{k_{H}} \sin \theta_{1} \frac{V}{c},
$$

where $k=2 \pi f / c$. The modal amplitude is now

$$
\tilde{\Gamma}(x, y) \sim q\left(\frac{e^{i k_{H} r_{0}}}{\sqrt{k_{H} r_{0}}}+R\left(\theta_{s}\right) \frac{e^{i \tilde{k}_{H}\left(r_{1}+r_{2}\right)}}{\sqrt{\tilde{k}_{H}\left(r_{1}+r_{2}\right)}}\right) .
$$

The horizontal wavenumber correction is a weighted Mach number that changes both amplitude and phase of the reflected paths. For an initial frequency of $55 \mathrm{~Hz}$ and $c=1500 \mathrm{~m} / \mathrm{s}$, the difference in $k_{H}$ and $\tilde{k}_{H}$ is about $0.6 \mathrm{~km}^{-1}$. Calculations focus on a single wavefront modeled as a jump to describe effects of the frequency change.

\section{CURVED NONLINEAR INTERNAL WAVE FRONTS}

The Huygens' formulation for the HLM is useful when the NIW front is not straight. Instead of the front along $y=y_{F}$ in Fig. 3(a), the front is $y=f(x)$ as in Fig. 3(d), and in formulas $r_{1}, r_{2}, R\left(\theta_{1}\right), \theta_{1}$ and $\theta_{2}$ are replaced by $\bar{r}_{1}, \bar{r}_{2}, \bar{R}\left(\bar{\theta}_{1}\right)$, $\bar{\theta}_{1}$, and $\bar{\theta}_{2}$. Source and receiver locations are in Sec. III. The angles $\bar{\theta}_{1}$ and $\bar{\theta}_{2}$ are between $\bar{r}_{1}$ and $\bar{r}_{2}$ and the line tangent to the reflector at any point $(\bar{x}, \bar{y})$ on the front. The incident and reflected rays become

$$
\bar{r}_{1}\left(\bar{\theta}_{1}\right)=\sqrt{\bar{x}^{2}+\left(\bar{y}-y_{F}\right)^{2}}=\frac{\bar{x}+m(\bar{x})\left[\bar{y}-y_{F}\right]}{\cos \bar{\theta}_{1} \sqrt{m(\bar{x})^{2}+1}},
$$

and

$$
\begin{aligned}
\bar{r}_{2}\left(\bar{\theta}_{1}\right) & =\sqrt{(L-\bar{x})^{2}+(d-\bar{y})^{2}} \\
& =\frac{[L-\bar{x}]+m(\bar{x})[d-\bar{y}]}{\cos \bar{\theta}_{2}\left(\bar{\theta}_{1}\right) \sqrt{m(\bar{x})^{2}+1}},
\end{aligned}
$$

where $m(\bar{x})$ is the slope of the line tangent to the front $\bar{y}=f(\bar{x})$. 
The reflected wave may be expressed using Huygens' Principle via Eq. (18). The equation for amplitude becomes

$$
\begin{aligned}
\bar{\Gamma}(L, d) \sim & q_{H} \frac{e^{i k_{H} r_{0}}}{\sqrt{k_{H} r_{0}}}+i k_{H} \frac{\psi\left(z_{s} ; 0,0\right)}{\rho\left(z_{s}\right)} \\
& \times \int_{0}^{\infty} \bar{R}\left(\bar{\theta}_{1}\right) \bar{C}\left(\bar{\theta}_{1}, \bar{\theta}_{2}\right) \frac{e^{i k_{H}\left(\bar{r}_{1}+\bar{r}_{2}\right)}}{\sqrt{k_{H}^{2} \bar{r}_{1} \bar{r}_{2}}} d x,
\end{aligned}
$$

where the obliquity factor is now

$$
\bar{C}\left(\bar{\theta}_{1}, \bar{\theta}_{2}\right)=\frac{\cos \left(\frac{\pi}{2}-\bar{\theta}_{1}\right)+\cos \left(\frac{\pi}{2}-\bar{\theta}_{2}\right)}{2} .
$$

The major contribution to the integral in Eq. (39) occurs at $\bar{\theta}_{1}=\bar{\theta}_{2}=\bar{\theta}_{s}$ according to stationary phase. Using Eqs. (37) and (38) gives the relation

$$
\frac{\bar{x}+m(\bar{x})\left[\bar{x}-y_{F}\right]}{\bar{r}_{1}}=\frac{[L-\bar{x}]+m(\bar{x})[d-\bar{x}]}{\bar{r}_{2}} .
$$

Equation (41) is equivalent to the requirement that the angles are equal and can be solved for the stationary point $\left(\bar{x}_{s}, \bar{y}_{s}\right)$. The integral term in Eq. (39) is denoted $\Gamma_{R}(L, d)$ and becomes

$$
\bar{\Gamma}_{R}(L, d) \sim q \bar{R}\left(\bar{\theta}_{s}\right) \frac{e^{i k_{H}\left(\bar{r}_{1}+\bar{r}_{2}\right)}}{\sqrt{k_{H}\left(\bar{r}_{1}+\bar{r}_{2}\right)}} \sqrt{\frac{\sin ^{2} \bar{\theta}_{s}}{\sin ^{2} \bar{\theta}_{s}+\chi\left(\bar{x}_{s}, \bar{y}_{s}\right)}},
$$

where $\chi\left(\bar{x}_{s}, \bar{y}_{s}\right)$ is the correction term introduced into Eq. (20) by front curvature. An expression for $\chi\left(\bar{x}_{s}, \bar{y}_{s}\right)$ is

$$
\begin{aligned}
\chi\left(\bar{x}_{s}, \bar{y}_{s}\right)= & m^{2}\left(1-\frac{\left(\bar{x}_{s}+m\left(\bar{y}_{s}-y_{F}\right)\right)^{2}}{\bar{r}_{1}^{2}\left(m^{2}+1\right)}\right) \\
& +\frac{M}{m}\left(\frac{\bar{r}_{1}\left(L-\bar{x}_{s}\right)-\bar{r}_{2} \bar{x}_{s}}{\left(\bar{r}_{1}+\bar{r}_{2}\right)}\right),
\end{aligned}
$$

where $M=f^{\prime \prime}\left(\bar{x}_{s}\right)$ is the approximate curvature of the front at the stationary point. For the formulation in Sec. III A, a straight front has $m=0$ and $M=0$, and Eq. (42) reduces to Eq. (20). For a straight tilted front $M=0$ while $m \neq 0$.

Although Eq. (43) holds for any continuous wavefront shape $f(x)$, this study focuses on circular fronts with

$$
f^{ \pm}(x)=y_{F} \pm\left(-\sigma+\sqrt{\sigma^{2}-x^{2}}\right),
$$

where $\sigma$ is the radius of curvature of the NIW. Equation (44) gives $m(\bar{x})= \pm \bar{x} / \sqrt{\sigma^{2}-\bar{x}^{2}}$, and the root depends on the orientation of the front. For a front in negative cross range relative to the source, the plus root corresponds to a concave front as in Fig. 3(d), and the minus root to a convex front. The maximum curvature considered is $\sigma=5 \mathrm{~km}$, which is consistent with SAR images in Ref. 2 as well as data from the SW06 experiment. ${ }^{30}$

The reflection coefficient $\bar{R}\left(\bar{\theta}_{s}\right)$ differs by a factor from the straight case although its derivation requires the same conditions on pressure and velocity. The stationary phase condition on the angle implies that

$$
\begin{aligned}
\bar{R}\left(\bar{\theta}_{s}\right) & =\left(\frac{\sin \bar{\theta}_{s}-i \sqrt{\cos ^{2} \bar{\theta}_{s}-v^{2}}}{\sin \bar{\theta}_{s}+i \sqrt{\cos ^{2} \bar{\theta}_{s}-v^{2}}}\right) e^{i k_{H} \bar{y} \sin \bar{\theta}_{s}} \\
& =R\left(\bar{\theta}_{s}\right) e^{i k_{H} \bar{y} \sin \bar{\theta}_{s}},
\end{aligned}
$$

where the phase change in Eq. (45) arises from the incident ray hitting the curved front. The range of angles where only reflection occurs is the same as in Sec. III A. Although the critical angle $\bar{\theta}_{s}$ has not changed, it now depends on the slope of the front, so the region where strong patterns occur changes with $\sigma$. As in Sec. III A, and using Eqs. (37) and (38), a pair of inequalities hold:

$$
v \geq \frac{m \bar{x}-\left(\bar{y}-y_{F}\right)}{\bar{r}_{1} \sqrt{m^{2}+1}},
$$

and

$$
v \geq \frac{m(L-\bar{x})-(d-\bar{y})}{\bar{r}_{2} \sqrt{m^{2}+1}} .
$$

These specify a more complicated expression for $d$ compared with a straight front, Eq. (26), for which examples had $\left|R\left(\theta_{s}\right)\right| \leq 1$ for $\theta_{s}$ less than 7-10 deg.

Table I shows examples of Eqs. (46) and (47), giving the maximum value of receiver cross range $d(m)$ where an HLM pattern extends from the front for the minus root of Eq. (44). Table II shows the same for the plus root in Eq. (44). Values are reported for a fixed $L=15 \mathrm{~km}$. As $s$ increases, the HLM range decreases. However, increasing curvature (decreasing $\sigma$ ) provides more opportunity for HLM patterns at $L=15 \mathrm{~km}$, and maximum $d$ values increase. For $s=200$ and $\sigma=100 \mathrm{~km}$, the maximum value is smaller compared to the trend in the rest of the row because the strong HLM region does not extend to the front.

In Table II, the maximum $d$ again decreases as $s$ increases but also decreases as $\sigma$ decreases (larger curvature). The latter behavior is opposite to that in Table I. In both cases, there are steeper angles with the front and fewer

TABLE I. Maximum distance $d(\mathrm{~m})$ between front and end of strong HLM region at $L=15 \mathrm{~km}$, depending on separation distances $s(\mathrm{~m})$ from source to front and front radius of curvature $\sigma(\mathrm{km})$. Maximum values are from Eqs. (46) and (47) and the minus root of Eq. (44). For $s=200$ and $400 \mathrm{~m}$ and $\sigma=100 \mathrm{~km}$, the region does not extend to the front [see Figs. 9(b) to 9(d)]. As a result these regions cannot sustain an HLM pattern, so values in Table I are adjusted to account for this phenomenon.

\begin{tabular}{lrrrrrr}
\hline \hline & \multicolumn{6}{c}{$\sigma(\mathrm{km})$} \\
\cline { 2 - 7 } $\mathrm{s}(\mathrm{m})$ & $\infty$ & 500 & 400 & 300 & 200 & 100 \\
\hline 200 & 1628 & 1776 & 1814 & 1879 & 2136 & 1839 \\
400 & 1428 & 1607 & 1632 & 1674 & 1829 & 2020 \\
600 & 1228 & 1349 & 1406 & 1430 & 1577 & 1925 \\
800 & 1028 & 1139 & 1177 & 1222 & 1320 & 1640 \\
1000 & 828 & 929 & 982 & 1010 & 1104 & 1403 \\
11200 & 628 & 745 & 785 & 826 & 920 & 1206 \\
1400 & 259 & 561 & 592 & 643 & 736 & 1009 \\
1600 & 220 & 379 & 422 & 464 & 578 & 848 \\
\hline \hline
\end{tabular}


TABLE II. Same as Table I, except using the plus root of Eq. (44). For $s$ large and $\sigma$ small enough, incidence angles are steep and strong HLM patterns are not seen.

\begin{tabular}{lrrrrrr}
\hline \hline & \multicolumn{7}{c}{$\sigma(\mathrm{km})$} \\
\cline { 2 - 7 } $\mathrm{s}(\mathrm{m})$ & $\infty$ & 500 & 400 & 300 & 200 & 100 \\
\hline 200 & 1628 & 1466 & 1428 & 1363 & 1138 & 756 \\
400 & 1428 & 1298 & 1226 & 1189 & 1070 & 735 \\
600 & 1228 & 1105 & 1064 & 1019 & 938 & 665 \\
800 & 1028 & 923 & 898 & 835 & 750 & 0 \\
1000 & 828 & 715 & 685 & 629 & 497 & 0 \\
1200 & 628 & 490 & 453 & 382 & 139 & 0 \\
1400 & 259 & 199 & 79 & 0 & 0 & 0 \\
1600 & 220 & 0 & 0 & 0 & 0 & 0 \\
\hline \hline
\end{tabular}

below critical. For large enough $s$ or small enough $\sigma$, no strong HLM pattern exists.

\section{EXAMPLES}

\section{A. Straight fronts}

In Sec. II B, it was hypothesized that a $\operatorname{sech}^{2}$ representation of an NIW may be approximated by a sound speed jump. Calculations using AMPE where the front is taken as a $\mathrm{sech}^{2}$ function are compared to results from the HLM model to illustrate that full field intensity patterns from a parabolic equation are essentially equivalent to HLM patterns. The Lloyd mirror-like patterns appear to occur outside the NIW at separation distances $s$ for which the incident acoustic propagation paths are within a critical angle.

The HLM predictions and AMPE calculations were compared for reduced TL sections along the $y$ axis at various distances $s$ between source and front. Figure 4(a) shows the AMPE result for $s=400 \mathrm{~m}$ over a $15 \mathrm{~km}$ range with $30 \mathrm{~dB}$ dynamic range. Figure 4(b) shows corresponding results for

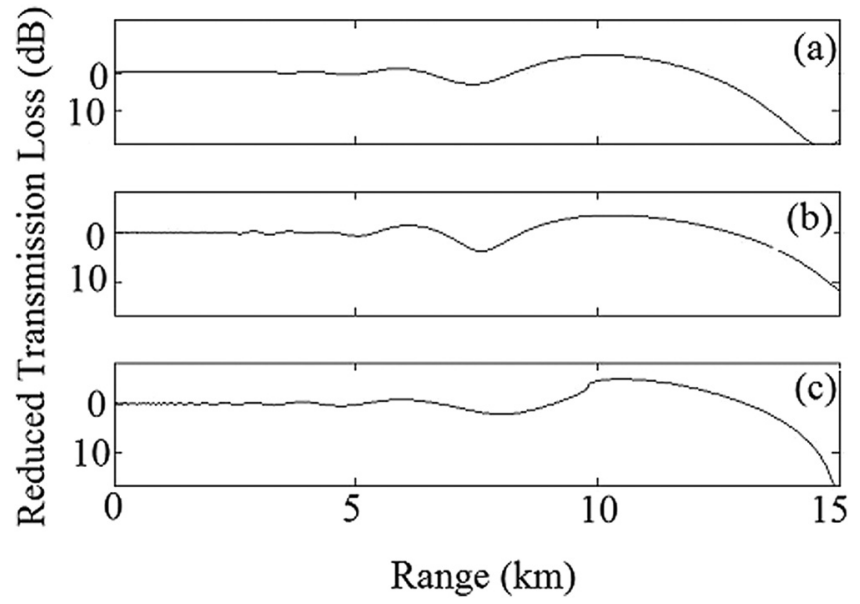

FIG. 4. Reduced TL along $y=0$ for a $15 \mathrm{~km}$ range and $s=400 \mathrm{~m}$. All plots have dynamic range of $30 \mathrm{~dB}$. (a) AMPE calculation for nonlinear internal wave environment. (b) Horizontal Lloyd mirror model calculation using the multilayer reflection coefficient $(l=3)$ and a boxcar with $\Delta c=5 \mathrm{~m} / \mathrm{s}$ and $w_{1}=500 \mathrm{~m}$. The modal amplitude is normalized by dividing by the first term in Eq. (21) for comparison with (a). (c) Same as (b) except calculations use a single $5 \mathrm{~m} / \mathrm{s}$ sound speed jump. The plots show good agreement in the number of fades and their placement.

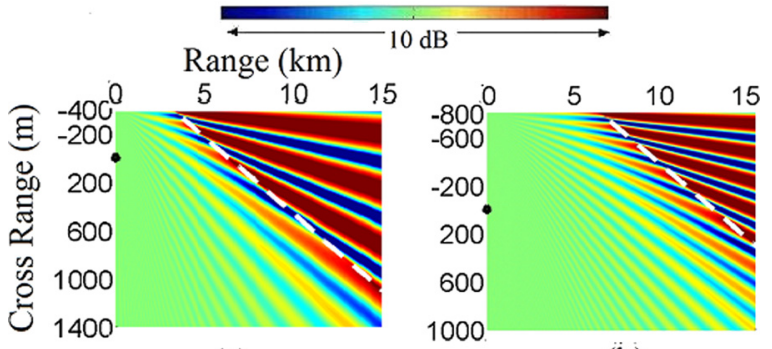

(a)

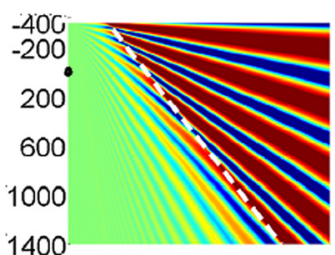

(c) (b)

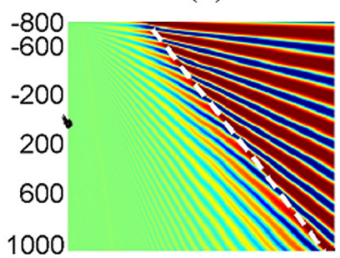

(d)
FIG. 5. (Color online) Reduced TL contours with calculations from Eq. (21) using sound speed change $\Delta c$ and source to front distance $s$ equal to (a) $12 \mathrm{~m} / \mathrm{s}$ and $400 \mathrm{~m}$, (b) $12 \mathrm{~m} / \mathrm{s}$ and $800 \mathrm{~m}$, (c) $24 \mathrm{~m} / \mathrm{s}$ and $400 \mathrm{~m}$, and (d) $24 \mathrm{~m} / \mathrm{s}$ and $800 \mathrm{~m}$. The modal amplitude is normalized by dividing through by the first term in Eq. (21), so calculations can also be fit to a $10 \mathrm{~dB}$ dynamic range for comparisons. The region to the right of the white dashed line is expected to have a strong HLM pattern via Eq. (26). Images neglect beam displacement. Regions of strong HLM patterns increase as $s$ decreases and as $\Delta c$ increases.

the HLM model without beam displacements using the multiple layer reflection coefficients given in Eqs. (30) and (31) for $l=3, w_{1}=500 \mathrm{~m}$, and $\Delta c=5 \mathrm{~m} / \mathrm{s}$, so that the boxcar and AMPE sech ${ }^{2}$ have comparable areas. Finally, Fig. 4(c) has calculations using the ESME reflection coefficient and a single $5 \mathrm{~m} / \mathrm{s}$ sound speed jump. Amplitudes of Figs. 4(b) and 4(c) are normalized by dividing out by the direct wave portion of Eq. (21) rather than the traditional $1 \mathrm{~m}$ reference value. This normalization implies a constant difference in TL compared to AMPE calculations, so calculations are fit to the same decibel dynamic range. Cases exhibit the same behavior in the number of fades and their approximate locations. As a result the AMPE calculations in Figs. 2(a) and 2(b) show generally good agreement with the HLM model for both NIW representations.

Figures 5(a) and 6(a) compare the jump and boxcar models with normalized amplitudes and contours are shown over a $10 \mathrm{~dB}$ range. The $s=400 \mathrm{~m}$ cases in Figs. 5(a) and 6(a) have a $\Delta c=12 \mathrm{~m} / \mathrm{s}$, and $w_{1}=200 \mathrm{~m}$ in the latter. In both, the familiar fanlike striation pattern occurs, and the number of beams and widths. The jump model produces more intense beams because the reflection from the second front may interfere destructively. Figure 6(b) shows contours for a front separation of $400 \mathrm{~m}$, and the beams are more intense than Fig. 6(a) due to the increase in area of the boxcar. Figure 6(c), with $\Delta c=6 \mathrm{~m} / \mathrm{s}$ and $w_{1}=400 \mathrm{~m}$ so the area agrees with the case in Fig. 6(a), has similar intensities. Finally, Fig. 6(d) with $w_{1}=800 \mathrm{~m}$ shows a prominent secondary reflection, which disrupts the interference pattern along the line where the beams fade to weaker TL values. The boxcar and jump models typically produce similar patterns until the separation of the jumps is about 10 times the acoustic wavelength and secondary reflections begin to 
(a)

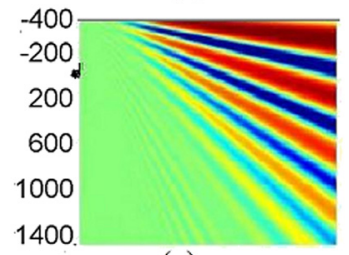

(c)

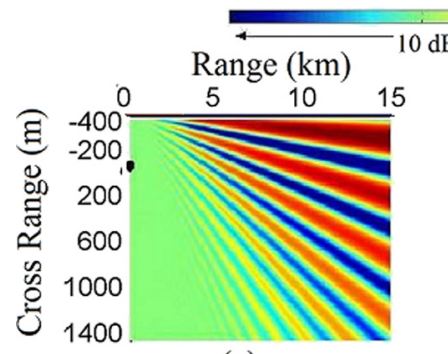

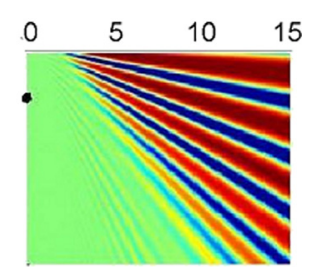

(b)

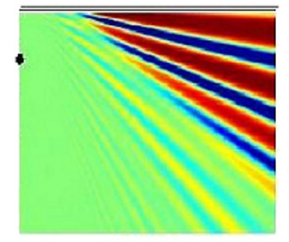

(d)
FIG. 6. (Color online) Same as Fig. 5 except reflection coefficient calculated using the multiple layer expressions in Eqs. (30) and (31) for two fronts. The value of $s$ is fixed at $400 \mathrm{~m}\left(y_{F}=-400 \mathrm{~m}\right)$. Magnitude of $\Delta c$ and NIW width $w_{1}$ are (a) $12 \mathrm{~m} / \mathrm{s}$ and $200 \mathrm{~m}$, (b) $12 \mathrm{~m} / \mathrm{s}$ and $400 \mathrm{~m}$, (c) $6 \mathrm{~m} / \mathrm{s}$ and $400 \mathrm{~m}$, and (d) $6 \mathrm{~m} / \mathrm{s}$ and $800 \mathrm{~m}$. In (d) $w_{1}$ gets large enough such that secondary reflections are visible.

appear. The effects from a range of NIW widths generally appear to be small. Consequently, for the regime where a typical NIW has 200-400 m width, other than the change in pattern intensity, the jump-front model captures relevant features of both boxcar and AMPE calculations. This simplification is used for further calculations with a single NIW.

Both Figs. 5(a) and 5(b) represent the NIW as a sound speed jump of $12 \mathrm{~m} / \mathrm{s}$, with 5 (b) having source-front distance $s=800 \mathrm{~m}$. Along the dashed line enclosing the region of strong HLM patterns from Eq. (26), a ripple is visible as the interference pattern transitions to weaker variations in TL levels. The ripple results from the kink in the reflection coefficient at the critical angle and corresponds to regions where beam displacement is predicted. For fronts further from the source, the number of beams increases and their widths decrease, and the region of strong interference patterns decreases as well. These results from the HLM model compare well with the classical Lloyd mirror using the Rayleigh reflection coefficient. The maximum number of beams for different front positions, using the same approach as in Refs. 27 and 31 , is very close with that from the model.

Figures 5(c) and 5(d) correspond to Figs. 5(a) and 5(b) except with sound speed jump of $24 \mathrm{~m} / \mathrm{s}$. For the former, the beam width and placement has not changed significantly, but the position of the dashed line is different, showing height intensity beams over a larger area compared to the latter. This arises because the critical angle for sound speed jump $24 \mathrm{~m} / \mathrm{s}$ is about $10 \mathrm{deg}$ and only $7 \mathrm{deg}$ in calculations for $\Delta c=12 \mathrm{~m} / \mathrm{s}$.

For multiple waves, the reflection coefficient Eqs. (30) and (31) are used to examine the case of two boxcarmodeled waves, $l=5$. Having seen the dependence on front position and on sound speed jump associated with a single NIW, $s=400 \mathrm{~m}$ and $\Delta c=12 \mathrm{~m} / \mathrm{s}$ are fixed to explore effects from wave spacing. Figure 7 shows calculations with two waves with pairs of fronts representing a NIW spaced $100 \mathrm{~m}$

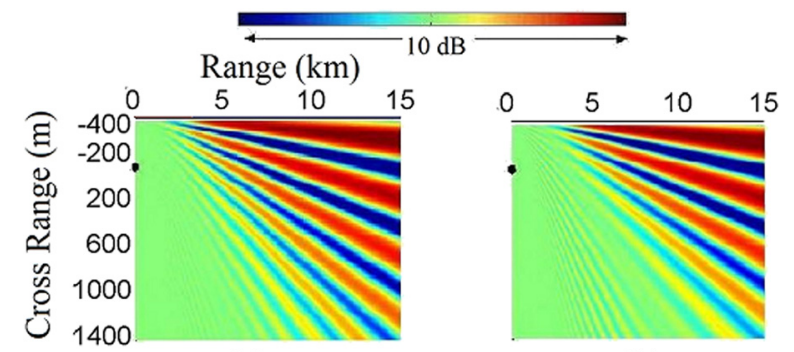

(a)

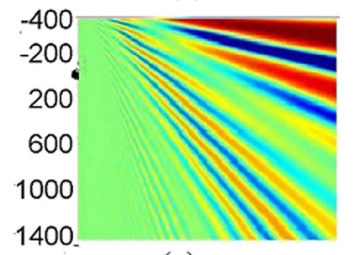

(c)

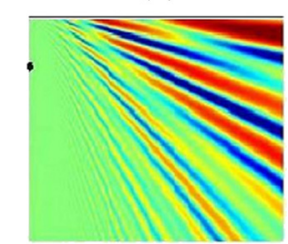

(d)

FIG. 7. (Color online) Same as Fig. 6, except four fronts simulate two waves in a train. Widths $w_{1}$, and $w_{3}$ of NIWs are $100 \mathrm{~m}$ and all sound speed jumps are $12 \mathrm{~m} / \mathrm{s}$. Spacing between the two waves $w_{2}$ is (a) $25 \mathrm{~m}$, (b) $100 \mathrm{~m}$, (c) $350 \mathrm{~m}$, and (d) $820 \mathrm{~m}$. Prominent beam interference is seen for large wave spacings.

apart so secondary reflections from the back of the boxcar are not present. Figure $7(a)$ is a limiting case in which $w_{2}=25 \mathrm{~m}$ so NIWs are close enough to be effectively one boxcar and the TL is very close to Fig. 6(a). As $w_{2}$ increases beyond $100 \mathrm{~m}$ [Fig. 7(b)], the second wave effects become more prominent. For example, in Fig. 7(c) an out of phase spacing with $w_{2}=350 \mathrm{~m}$ shows another appearance of secondary reflection. In Fig. $7(\mathrm{~d})$ with $w_{2}=820 \mathrm{~m}$, the two interruptions in the pattern are a clear superposition of interference patterns from the two waves.

Effects of the Doppler shift from an NIW moving at constant speed are seen in Fig. 8. The snapshots are all taken with the source and front separated by $100 \mathrm{~m}$ and NIWs

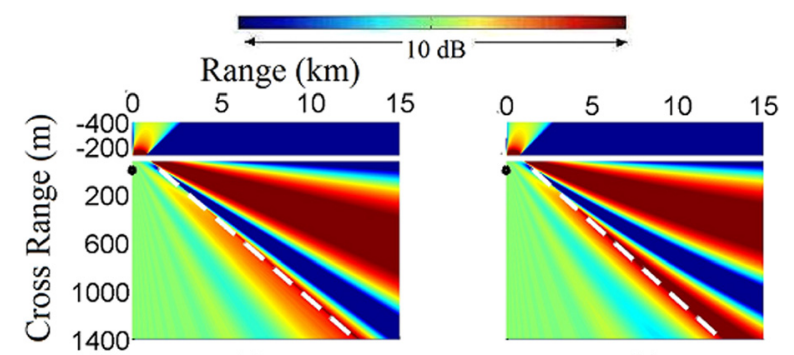

(a)

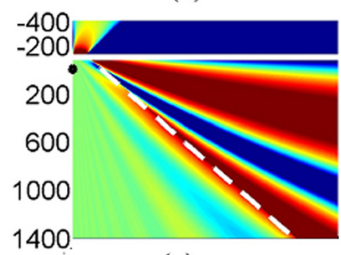

(c) (b)

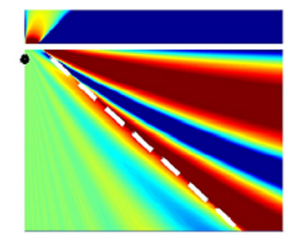

(d)

FIG. 8. (Color online) Same contours as Fig. 5 except fronts are moving. The region to the right of the white dashed line is expected to have a strong HLM pattern via Eq. (26). Snapshots taken when the front, represented by the solid white line, is at $-100 \mathrm{~m}$ in cross range, and the source and front are $100 \mathrm{~m}$ apart. Range is $15 \mathrm{~km}$ and total cross range is $1800 \mathrm{~m}$. The speed $V$ of the NIW is (a) $0 \mathrm{~m} / \mathrm{s}$, (b) $0.6 \mathrm{~m} / \mathrm{s}$, (c) $1.3 \mathrm{~m} / \mathrm{s}$, and (d) $2 \mathrm{~m} / \mathrm{s}$. As $V$ increases more strong beams are observed and they begin to curve. 


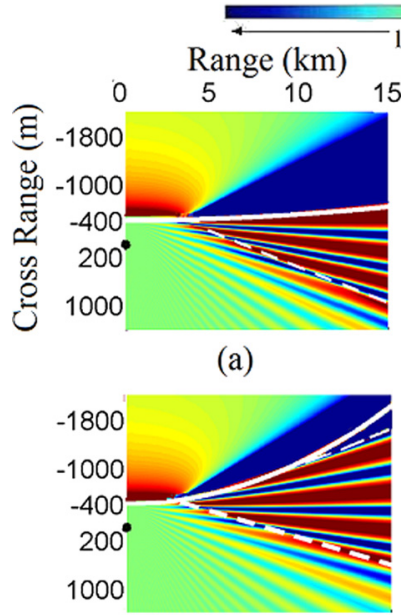

(c)

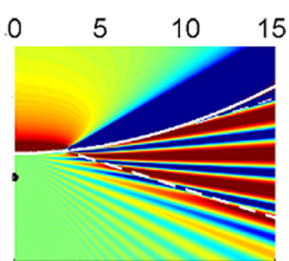

(b)

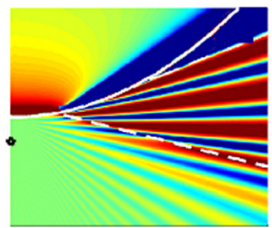

(d)
FIG. 9. (Color online) Same contours as Fig. 5. Calculations use curved fronts with $\Delta c=12 \mathrm{~m} / \mathrm{s}$ and the convex wavefront shape $f^{-}(x)$ of Eq. (44). The distance $s$ from top of the internal wave front to source is $400 \mathrm{~m}$. The NIW front is denoted by the solid white line, and the white dashed lines enclose the region expected for a strong HLM pattern from the constraints of Eqs. (46) and (47). Images neglect beam displacement. Note cross range extends to $3600 \mathrm{~m}$. The radius of curvature of the front is (a) $500 \mathrm{~km}$, (b) $100 \mathrm{~km}$, (c) $70 \mathrm{~km}$, and (d) $50 \mathrm{~km}$. As radius of curvature decreases, strong HLM patterns are seen over larger regions.

moving with speeds between zero and $2 \mathrm{~m} / \mathrm{s}$. Calculations for the same source and front separation eliminate the nontrivial effects from different separations. A stationary front in Fig. 8(a) has one strong straight beam and with increasing speed a second strong beam appears in Fig. 8(b). Curvature of the beams is seen for speeds above $1 \mathrm{~m} / \mathrm{s}$ in Figs. 8(c) and 8 (d) due to the $\sin \left(\theta_{1}\right)$ correction in the horizontal wavenumber in Eq. (35). Section III C shows the resulting change in the horizontal wavenumber, of about $0.6 \mathrm{~km}^{-1}$, that modifies the intensity and shapes.

\section{B. Curved fronts}

Effects of NIW front radius of curvature on HLM patterns are illustrated in Figs. 9 and 10 with sound speed increase of $12 \mathrm{~m} / \mathrm{s}$ and $s=400 \mathrm{~m}$. For $\sigma \geq 5 \mathrm{~km}$, the term $M$ containing curvature in Eq. (43) has negligible contributions for parameter values of interest. Therefore, it is the term containing $m$, representing front tilt at the interaction point, which is relevant.

Both figures show $\sigma$ values from 500 to $50 \mathrm{~km}$, and the dashed lines enclosing regions of strong HLM pattern are found from the constraints in Eqs. (46) and (47). Figure 9, on a cross range scale from -2200 to $1400 \mathrm{~m}$, has the source oriented before the front so that the NIW appears convex, and the minus root of Eq. (44) is chosen. As curvature increases in Fig. 9, the beams spread slightly. A low intensity region on the source side appears in Fig. 9(b) near the front when $L \geq 12 \mathrm{~km}$. Receivers in this region produce no HLM pattern without direct or reflected paths. This region visibly increases in area in Figs. 9(c) and 9(d). Thus patterns produced by convex fronts are similar to straight fronts except for the additional low intensity region.

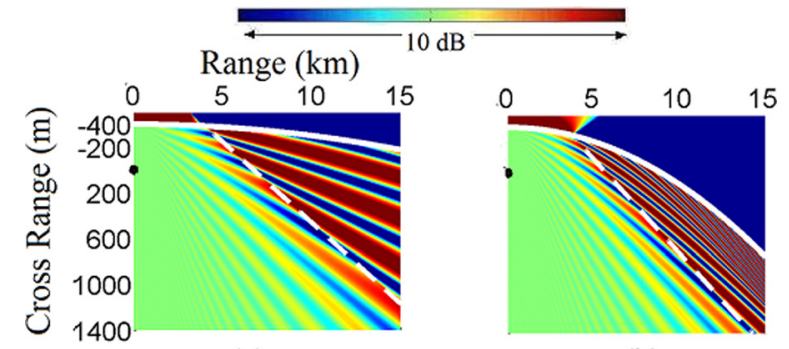

(a)

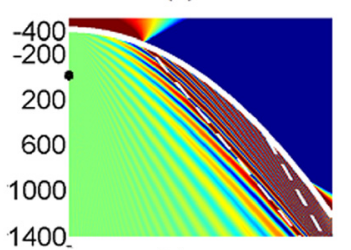

(c)

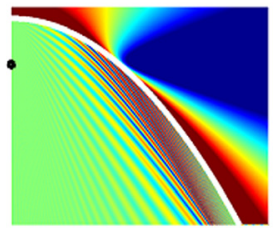

(d)

FIG. 10. (Color online) Same as Fig. 9, except using the concave wavefront shape $f^{+}(x)$ of Eq. (44). Total cross range is $1900 \mathrm{~m}$. Frontal radius of curvature is (a) $500 \mathrm{~km}$, (b) $100 \mathrm{~km}$, (c) $70 \mathrm{~km}$, and (d) $50 \mathrm{~km}$. As radius of curvature decreases, strong HLM patterns are seen over smaller regions.

Figure 10 shows a source oriented inside the front, so the NIW appears concave. At $\sigma=500 \mathrm{~km}$ in Fig. 10(a), the contours show little influence of curvature. As $\sigma$ increases, the beams become considerably narrower and occur over a smaller area. Increasing curvature brings fewer receiver positions with incident angles below $7 \mathrm{deg}$, so the area with total internal reflection and strong HLM pattern decreases, as in Fig. 10(b) with $\sigma=100 \mathrm{~km}$. In Fig. 10(c) with $\sigma=70 \mathrm{~km}$, not only does the region decreases in size, but also a second dashed line appears because fewer incident angles for the front position $y_{F}=-400 \mathrm{~m}$ satisfy the critical angle condition. Consequently, the phenomenon of an HLM window arises. For $\sigma=50 \mathrm{~km}$ in Fig. 10(d), no dashed lines appear because incident angles for these range and cross range positions forbid strong HLM patterns due to the curvature. Although NIW curvature radii are observed as low as $5 \mathrm{~km}$, the critical angles forbid HLM patterns, as suggested by the results in Table II. In contrast to convex fronts, NIW fronts that appear concave influence the critical angle and decrease regions of strong HLM patterns to eventually produce HLM windows.

\section{CONCLUSIONS}

Interference patterns in horizontal planes can be produced by the propagation of a single mode in the presence of a nonlinear internal wave and have a direct analogy with classical Lloyd mirror patterns that occur in vertical planes. ${ }^{1}$ These horizontal Lloyd mirrors (HLMs) arise from a direct path and a refracted path through a NIW front and have been observed recently in experimental data. ${ }^{15}$ Three representations for the NIWs are considered: soliton solutions of the $\mathrm{KdV}$ equation, boxcar approximations consisting of two sound speed jumps, and single sound speed jumps. Comparisons are made among solutions for these cases, using an adiabatic mode parabolic equation method and asymptotic solution formulas from Huygens' Principle and the 
stationary phase method. The results show the advantages and limitations of using a single jump model, which is used for most subsequent calculations in this paper.

When modeling the front as a single sound speed jump, it was seen that as separation between source and front increases beam widths and regions of strong interference patterns decrease. Also, with increases in the sound speed jump, which can be associated with NIW amplitude, the region of strong patterns broadens. Multiple fronts not only influence beam intensity but also introduce secondary reflections. Two identical waves are also discussed. Waves closely spaced behave as one NIW, and as separation increases, secondary reflections appear that show a superposition of patterns from the different waves. Additionally, wave speed can change HLM patterns. For speeds of about $1 \mathrm{~m} / \mathrm{s}$, the number of strong beams increases, and as the speed tends toward $2 \mathrm{~m} / \mathrm{s}$ the beams become curved.

Huygens' Principle works well for extensions incorporating curved fronts. Results are shown for convex and concave fronts relative to a source. Convex fronts cause a shadow region in areas where there are no direct or reflect paths to the receiver. Concave fronts decrease regions of strong HLM patterns and eventually show an HLM window along the front as curvature increases. It was seen that strong HLM patterns do not occur in the region of interest for $\sigma \leq 50 \mathrm{~km}$. Investigating the HLM parameter dependence of multiple and moving fronts with curvature, along with the use of multiple modes, deserves investigation.

\section{ACKNOWLEDGMENTS}

The authors thank the Office of Naval Research for funding this work. Additionally, the first author is supported through an ONR Ocean Acoustics Traineeship. The authors also thank Dr. John Colosi and Dr. Ying-Tsong Lin for useful discussions.

${ }^{1}$ J. F. Lynch, J. C. Colosi, G. Gawarkiewicz, T. F. Duda, A. D. Pierce, M. Badiey, B. Katsnelson, J. E. Miller, W. Siegmann, C. Chiu, and A. Newhall, "Consideration of fine-scale coastal oceanography and 3-D acoustics effects for the ESME sound exposure model," IEEE J. Ocean. Eng. 31, 33-49 (2006).

${ }^{2}$ C. R. Jackson and J. R. Apel, "An atlas of internal solitary-waves and their properties," Technical Report prepared under contract with ONR N00014-98-C-0238, Global Ocean Associates (2002), www.internalwaveatlas.com (date last viewed 2/10/11).

${ }^{3}$ J. R. Apel, L. A. Ostrovsky, Y. A. Stepanyants, and J. F. Lynch, "Internal solitons in the ocean," Report MERCIRA0695, Appl. Phys. Lab., Johns Hopkins University (1995).

${ }^{4}$ J. A. Colosi, R. C. Beardsley, J. F. Lynch, G. Gawarkiewicz, C. S. Chiu, and A. Scotti, "Observations of nonlinear internal waves on the outer New England continental shelf during the summer shelfbreak PRIMER study," J. Geophys. Res. 106, 9587-9601 (2001).

${ }^{5}$ T. F. Duda and J. C. Preisig, "A modeling study of acoustic propagation through moving shallow water solitary wave packets," IEEE J. Ocean. Eng. 24, 16-32 (1999).

${ }^{6}$ J. F. Lynch and D. Tang, "Overview of Shallow Water 2006 JASA-EL special issue papers," J. Acoust. Soc. Am. 124, EL63-EL65 (2008).
${ }^{7}$ J. C. Preisig and T. F. Duda, "Coupled acoustic mode propagation through continentalshelf internal solitary waves," IEEE J. Ocean. Eng. 22, 256-269 (1997)

${ }^{8}$ D. Rubenstein, "Observations of cnoidal internal waves and their effect on acoustic propagation in shallow water," IEEE J. Ocean Eng. 24, 346-357 (1999).

${ }^{9}$ B. G. Katsnelson and V. G. Petnikov, Shallow-Water Acoustics (Springer, Berlin, 2002), pp. 55-62.

${ }^{10}$ S. D. Frank, M. Badiey, J. F. Lynch, and W. L. Siegmann, "Experimental evidence of three-dimensional acoustic propagation caused by nonlinear internal waves," J. Acoust. Soc. Am. 118, 723-734 (2005).

${ }^{11}$ M. Badiey, B. G. Katsnelson, J. F. Lynch, S. Pereselkov, and W. L. Siegmann, "Measurement and modeling of three-dimensional sound intensity variations due to shallow-water internal waves," J. Acoust. Soc. Am. 117, 613-625 (2005).

${ }^{12}$ R. Oba and S. Finette, "Acoustic propagation through anisotropic internal wave fields: Transmission loss, cross-range coherence, and horizontal refraction,” J. Acoust. Soc. Am. 111, 769-784 (2002).

${ }^{13}$ M. Badiey, B. G. Katsnelson, J. F. Lynch, and S. Pereselkov, "Frequency dependence and intensity fluctuations due to shallow water internal waves," J. Acoust. Soc. Am. 122, 747-760 (2007).

${ }^{14}$ W. M. Carey, "Lloyd's mirror-image interference effects," Acoust. Today 3, 14-20 (2009).

${ }^{15}$ B. G. Katsnelson, V. Grigorev, M. Badiey, and J. F. Lynch, "Horizontal interference structure of the sound field in the presence of moving internal waves and estimation of angle of horizontal refraction (A)," J. Acoust. Soc. Am. 128, 2334 (2010).

${ }^{16}$ M. Badiey, B. G. Katsnelson, Y. T. Lin, and J. F. Lynch, "Acoustic multipath arrivals in the horizontal plane due to approaching nonlinear internal waves." J. Acoust. Soc. Am. 129, EL141-EL147 (2011).

${ }^{17} \mathrm{M}$. Collins, "The adiabatic mode parabolic equation," J. Acoust. Soc. Am. 94, 2269-2278 (1993).

${ }^{18}$ J. Apel, M. Badiey, C. Chiu, S. Finette, R. Headrick, J. Kemp, J. Lynch, A. Newhall, M. Orr, B. Paseward, D. Tielbürger, A. Turgut, K. V. der Heydt, and S. Wolf, "An overview of the 1995 SWARM shallow-water internal wave acoustic scattering experiment," IEEE J. Ocean. Eng. 22, 465-500 (1997)

${ }^{19}$ D. Rouseff, D. Tang, K. L. Williams, and Z. Wang, "Mid-frequency sound propagation through internal waves at short range with synoptic oceanographic observations," J. Acoust. Soc. Am. 124, EL73-EL77 (2008).

${ }^{20}$ T. F. Duda, "Acoustic mode coupling by nonlinear internal wave packets in a shelfbreak front area," IEEE J. Ocean. Eng. 29, 118-125 (2004).

${ }^{21}$ D. Tielbürger, S. Finette, and S. N. Wolf, "Acoustic propagation through an internal wave field in a shallow water waveguide," J. Acoust. Soc. Am. 101, 789-808 (1997).

${ }^{22}$ B. Katsnelson, V. Grigorev, and J. F. Lynch, "Intensity fluctuations of midfrequency sound signals passing through moving nonlinear internal waves," J. Acoust. Soc. Am. 124, EL78-EL84 (2008).

${ }^{23}$ R. B. Evans, "A coupled mode solution for acoustic propagation in a waveguide with step-wise depth variations of a penetrable bottom," J. Acoust. Soc. Am. 73, 188-195 (1983)

${ }^{24} \mathrm{R}$. Burridge and H. Weinberg, "Horizontal rays and vertical modes," in Wave Propagation and Underwater Acoustics,"edited by J. Keller and J. S. Papadakis, (Springer, Berlin, 1997), pp. 86-152.

${ }^{25} \mathrm{~J}$. M. Stone, Radiation and Optics, An Introduction to the Classical Theory (McGraw-Hill, New York, 1963), pp. 247-255.

${ }^{26} \mathrm{M}$. Brekhovskikh and Y. P. Lysanov, Fundamentals of Ocean Acoustics (Springer-Verlag, New York, 1982), pp. 76-90.

${ }^{27}$ F. B. Jensen, W. A. Kuperman, M. B. Porter, and H. Schmidt, Computational Ocean Acoustics (Springer, Berlin, 2000), pp. 14-17 and 44-49.

${ }^{28}$ L. M. Brekhovskikh, Waves in Layered Media (Springer-Praxis, New York, 2001), pp. 265-277.

${ }^{29}$ A. Gjurchinovski, "The Doppler effect from a uniformly moving mirror," Eur. J. Phys. 26, 643-646 (2005).

${ }^{30}$ J. F. Lynch, Y. T. Lin, T. F. Duda, and A. E. Newhall, "Acoustic ducting, shadowing, refraction and dispersion by curved non-linear internal waves in shallow water," IEEE J. Ocean. Eng. 35, 12-27 (2010).

${ }^{31}$ I. Tolstoy and C. S. Clay, Ocean Acoustics (AIP Press, New York, 1987), pp. 98-105. 